\title{
DAS TESTAMENT ALS EINE TEXTSORTE. EINE LINGUISTISCHE ANALYSE DES TESTAMENTS DES GALIZISCHEN ADLIGEN HERMAN DE BRUNICKI AUS DEM JAHRE 1835
}

\begin{abstract}
Testament as a text type
The aim of the article is to show the specific characteristics of the Galician legal language in the first half of the 19th century. The article analyses the 1835 will of Baron Herman de Brunicki. First, the pattern of the will, its structure and function are examined. Further on, the authors focus on the graphematic, lexical and syntactic levels of the will.
\end{abstract}

KeYwords: text type, testament, will, linguistic history, Galicia

\section{Einleitendes}

Der Beitrag verfolgt das Ziel, die Spezifik des Sprachgebrauchs im Rechtsbereich in der ersten Hälfte des 19. Jahrhunderts in Galizien zu reflektieren. Am Beispiel einer Fachtextsorte wie des Testaments sollen sowohl ihre textsortenspezifischen Besonderheiten als auch die sprachlichen Phänomene der galizischen Rechtssprache analysiert werden. Es handelt sich dabei um die Analyse der graphematischen, lexikalischen und syntaktischen Ebene anhand dieser Fachtextsorte. Bezeichnungen wie Rechtssprache und Verwaltungssprache, die unter den Begriffen Fachsprache und Fachtextsorten subsumiert werden können, werden hier synonym verwendet. Fachsprachliche Textsorten im Rechtsbereich definieren wir in diesem Falle als sprachlichen Vorrat für kommunikative Problemlösungen in der begrifflichen, normativ-regulativen Bestimmung rechtsspezifischer Gegenstände. Darüber hinaus gehen wir im Sinne von Wiktorowicz (2011) davon aus, dass die Textsortengeschichte eine Mentalitätsgeschichte darstellt.

Waldemar Czachur - Uniwersytet Warszawski, Warszawa, waldemar.czachur@uw.edu.pl

Agnieszka Zimmer - Uniwersytet Warszawski, Warszawa, a.zimmer@uw.edu.pl 


\section{Desiderate und Forschungsinteressen}

Die Frage nach dem Wesen der Fachsprache, darunter nach der Spezifik der Textsorten im Bereich der Verwaltung, des Rechts oder der Institutionen, war in der germanistischen wie auch der polonistischen Forschung oft Gegenstand linguistischer synchroner, diachroner sowie kontrastiver Analysen (vgl. Hoffmann, 1989; Becker-Mrotzek, 1999; Becker-Mrotzek \& Scherner, 2000; Piętkowa, 2000; Malinowska, 2001; Wyrwas, 2002; Rzeszutko, 2003; Wojtak, 2004; Czachur, 2007, 2008). Auch die deutsche Kanzleisprache auf dem Gebiet des heutigen Polen wurde intensiv untersucht, wie z. B. die Krakauer Kanzleisprache, darunter Testamente und Urkunden (vgl. Wiktorowicz, 2011, 2012; Kaleta-Wojtasik, 2001, 2004), die Thorner Kanzleisprache (vgl. Grabarek, 1984), das Kanzleischrifttum des 15. und 16. Jahrhunderts aus Namslau/Namysłów, Brieg/Brzeg, Neisse/Nysa und Leobschütz/Głubczyce (Bogacki, 2009), die ältesten Stadtbücher der niederschlesischen Stadt Sprottau/Szprotawa aus dem 16. Jahrhundert (Biszczanik, 2013) und die Kanzleisprache des Herzogtums Teschen (Chromik, 2010). Wegweisend für die Erforschung der Textsorte Testament, auch aus diachroner und historischer Perspektive, sind die Arbeiten von Żmigrodzka (1997), Spáčilová (2000), Kaleta-Wojtasik (2001), Bieberstedt (2007) und Wiktorowicz (2011).

Die Sprachwissenschaftler, darunter auch die polnischen Germanisten, die sich mit der Sprachgeschichte und vor allem mit den „deutschen Spuren“ im heutigen Polen beschäftigten, haben die Sprache, auch die Verwaltungs- und Rechtssprache, in Galizien kaum zum Gegenstand ihrer Reflexionen herangezogen. Bis auf die Arbeiten von Feret $(2013,2014)$ und Cwanek-Florek $(2015,2018)$ zum deutsch-polnischen Sprachkontakt und zu den daraus entstandenen sprachlichen Phänomenen wie Entlehnungen in Galizien sind in diesem Bereich keine Leistungen vorzuweisen. Umso interessanter erscheint in diesem Zusammenhang der Band von Hanus und Büttner Galizien als Kultur- und Gedächtnislandschaft im kultur- und sprachwissenschaftlichen Diskurs (2015) als eine wichtige Arbeit.

Mit diesem Beitrag möchten wir diese Lücke schließen oder zumindest verkleinern und zugleich aber auch die allzu hohen Erwartungen etwas dämpfen, denn es handelt sich hier um einen Versuch, zum einen eine diachrone Analyse der Rechtssprache anhand einer konkreten Textsorte und zum anderen die Spezifik der deutschen Sprache in Galizien in der ersten Hälfte des 19. Jahrhunderts zu erforschen. Als Grundlage fungiert die Abschrift des Testaments von Hermann Freiherr Brunicki aus dem Jahre 1835.

\section{Galizien - politischer und sprachlicher Hintergrund}

Galizien als habsburgisches Kronland, das offiziell Königreich Galizien und Lodomerien hieß, bestand von 1772 bis 1918 und fungierte als eine multiethnische, 
mehrsprachige und multikulturelle Landschaft (vgl. Besier \& Stokłosa, 2015). Mit der Teilung Polens wurde Galizien im Jahre 1772 zu einem deutsch verwalteten absolutistischen Staat, in dem auch der polnische Adel entmachtet wurde. Die deutsche Sprache wurde als Amtssprache, darunter auch als Verwaltungssprache, eingeführt. Neben der deutschen Sprache wurden auch das Polnische sowie das Ukrainische (Ruthenische) verwendet. Erst um 1868 begann wieder eine stärkere Polonisierung im öffentlichen Diskurs. Zu Recht stellen Besier \& Stokłosa (2015, S. 19) fest, dass es

[...] bisher [...] über Galizien in der ersten Hälfte des 19. Jahrhunderts, vor allem hinsichtlich der Beziehungen zwischen den verschiedenen Nationalitäten, kaum Untersuchungen [gibt]. Während für polnische Galizien-Forscher das Sprachenproblem im Wege stand (man muss deutsche Quellen lesen), haben deutsche und österreichische Forscher bislang kaum Interesse an Galizien gezeigt. Darum wäre es eigentlich an der Zeit, die Geschichte Galiziens in der ersten Hälfte des 19. Jahrhunderts - gerade hinsichtlich des multiethnischen Charakters dieser Region - neu zu bedenken.

Hinsichtlich der Forschung im Bereich der Sprachgeschichte, auch des Sprachkontakts in Galizien, soll, im Zusammenhang der drei Sprachen, die einen unterschiedlichen sozialen Status hatten, noch auf die symbolische Funktion der Sprachen verwiesen werden. Fellerer (2004, S. 54) schreibt dazu Folgendes:

Das Deutsche etwa als galizische Verwaltungssprache erfüllte nicht nur rein kommunikative Zwecke, sondern symbolisierte als Sprache der Dynastie das Haus Habsburg. Das Ruthenische als weiteres Beispiel diente nicht nur weiten Teilen der ostgalizischen Bauernschaft als Kommunikationsmittel, sondern symbolisierte bis weit in das 19. Jahrhundert konfessionelle und soziale Zugehörigkeit. Gleiches gilt für das Polnische, das u. a. als Symbol für den polnischen Adel und zugleich als Symbol für die katholische polnische Nation stand, die zeitweise gegen die österreichische Teilungsmacht kämpfte.

Insbesondere im Bereich der Verwaltung, also der Organisation des Staates und der rechtlichen Grundlagen, dominierte das Deutsche und beeinflusste mit den zahlreichen politischen Regulierungsmaßnahmen dezidiert das Sprachverhalten der galizischen Bevölkerung. Die Bevölkerungsstruktur sah in Galizien wie folgt aus: Etwa $50 \%$ der österreichischen Galizier waren Polen, $40 \%$ waren aus der Ukraine und $10 \%$ waren Juden.

Als konkretes Beispiel der Verwendung der deutschen Sprache im Bereich der Verwaltungs- und Justizsprache in Galizien gilt hier die Abschrift des Testaments von Hermann Freiherr Brunicki, dessen Vater Izaak Brunstein zum Christentum übergetreten war, um Güter in Galizien erwerben zu dürfen. Hermann Bruncki, geb. am 17.06.1776, verstorben am 20.04.1835 in Płazów, erwarb am 20.04.1821 die Güter in Ruda Różaniecka, Huta Różaniecka, Lubliniec Nowy und Lubliniec Stary, Żuków, Freifeld, Kosobudy, Krupiec und Płazów. Kurz vor seinem Tod setzte er das Testament auf, das die Grundlage dieser linguistischen Analyse sein wird. 


\section{Das Testament und sein Muster - der rechtliche und linguistische Kontext}

Das Wort Testament wurde im 15. Jh. mit der Einführung des römischen Rechts entlehnt. Nach Pfeifer (1993, S. 1427) geht das Wort auf das lat. testāmentum zurück, das seinerseits eine Bildung zu lat. testārī (testātus sum), bezeugen, bekunden, versichern, beweisen, seinen letzten Willen kundtun“ ist, dem wieder lat. testis „Zeuge“ zugrunde liegt. Griech. diathēkē ,Anordnung, letzter Wille, Vergleich, Vertrag' steht in der Kirchensprache für das durch Gott beschlossene (Altes Testament), dann (Neues Testament) durch Christus erneuerte Bündnis, den Gnadenbund mit den Menschen, sowie für die Schriften desselben. In der Vulgata wurde das griechische Wort mit dem kirchenlat. testāmentum wiedergegeben. Daraus wurde mhd. tëstamënt, Vertrag, Bündnis, Vermächtnis` entlehnt. Das heutige Deutsch unterscheidet zwischen zwei Bedeutungen des Wortes: ,letztwillige schriftliche Erklärung, in der jemand die Verteilung seines Vermögens nach seinem Tode festlegt' (auch in übertragenem Gebrauch, z. B. das politische Testament des großen Staatsmannes) und, Vertrag, Bund Gottes mit den Menschen' (vgl. DUDEN, 2006, S. 1674).

Das Testament ist, der rechtlichen Definition nach, eine einseitig getroffene Verfügung von Todes wegen eines voll Geschäftsfähigen, die jeder Zeit widerrufen werden kann. Ein Testament kann der zukünftige Erblasser nur alleine errichten (Napierała \& Plesiński, 2013, S. 373). Um gültig zu werden und zu bleiben, muss ein Testament einige Voraussetzungen erfüllen. Dazu gehören u. a. die Möglichkeit der selbstständigen Willenserklärung, das Bewusstsein der Vermögensverfügung sowie die schriftliche Form. Das Testament darf nur von einer Person geschrieben werden. Die meisten Rechtssysteme lassen gemeinschaftliche Testamente nicht zu. Ein den zwingenden Formvorschriften nicht genügendes Testament ist nichtig (Napierała \& Plesiński, 2013, S. 373). Diese Voraussetzungen sind im heutigen Rechtszustand sanktioniert und schriftlich in unterschiedlichen Gesetzbüchern fixiert, waren aber auch in früheren Perioden in der jeweiligen Form präsent. Dies betrifft vor allem das unbegrenzte Bewusstsein und den freien Willen des Erblassers. Das Prinzip eines Testaments war und bleibt dasselbe: Der Erblasser verfügt frei, ohne jeglichen Zwang und bei gesundem Bewusstsein, über das eigene Vermögen und verteilt das Erbe unter seine Erben. Eine darauf hinweisende Formulierung muss in einem Testament explizit erwähnt werden. Dies resultiert aus der allgemeinen Annahme, dass man sich dessen bewusst sein muss, was man tut, und dass jede nicht nur rechtliche Tätigkeit freiwillig ist, d. h., sie darf nicht durch Zwang, Einschüchterung, Erpressung o. Ä. beeinflusst sein.

Das Testament als Textsorte war bisher nur selten Gegenstand linguistischer Auseinandersetzungen, insbesondere für die Kanzleisprachen (vgl. Wiktorowicz, 2011; Spáčilová, 2000; Kaleta-Wojtasik, 2001; Bieberstedt, 2007, 2009). In Anlehnung an Wiktorowicz (2011, S. 155) gehen wir von folgendem textsortenlinguistischen Testamentsbegriff aus: 
Wenn man das Sprechen und Schreiben als eine Art soziale Handlung auffasst, dann müssen die Textsorten von diesem Standpunkt aus als Vollzug bestimmter Typen sozialer Handlungen verstanden werden. Aus der sozialen Handlung, bei der ein Stadtbürger vor seinem Tod über die Aufteilung seines Vermögens eine Entscheidung trifft, ergibt sich eine Sprachhandlung, die in Form eines relativ einheitlichen Textes im Stadtbuch festgehalten wird. Der einheitliche Typ sozialer Handlung führt zur Entstehung einer relativ einheitlichen Textsorte, denn das Testament ist eine Fixierung der letztwilligen Entscheidung über die Vermögensaufteilung eines Bürgers. Das konstante konstitutive Merkmal der Textsorte, Testament' ist die soziale Handlung, die man als Formulierung des letzten Willens bezeichnen kann.

Das Testament als eine Textsorte gilt hier als Instrument, mit der „eine Rechtshandlung vollzogen wird, deren volle Realisierung erst nach dem Tod des Verfassers des Testaments eintritt" (Wiktorowicz, 2011, S. 156). Spáčilová weist darauf hin, dass dabei drei Subjekte konstitutiv sind: der Testator, auch Testierer oder Erblasser genannt, der bei seinem Tode sein Erbe hinterlässt, dann der Erbnehmer als der Vermögensnachfolger des Erblassers sowie der Testamentsvollstrecker als vom Erblasser berufener Mensch, der letztwillige Anordnungen ausführen soll. Als ein konstantes Element muss man die Entscheidung über die Aufteilung des Vermögens durch den Testamentsautor ansehen. Denn in jedem Testament wird genau aufgelistet, wer was und auf welche Art und Weise bekommt.

Als eine traditionelle, stark formalisierte Urkunde weist das Testament viele rechtliche Eigenschaften auf. Als eine Rechtsurkunde fügt sich das Testament in eine sui generis Landschaft rechtlicher Texte ein und unterliegt konkreten, damit verbundenen Voraussetzungen, zu denen u. a. die höchstpräzise Sprache gehört. Weil viele rechtliche Zustände keine hundertprozentige Entsprechung haben, werden auch im Testament als einer Rechtsurkunde gewisse lateinische Formeln gebraucht, die die Situation genau und ohne jegliche Missverständnisse beschreiben.

Das Testament als Textsorte wird dem Texttyp Urkunde zugeordnet (vgl. Spáčilová, 2000), die drei Äußerungsteile (I-III) mit zwölf Textstrukturelementen umfasst (Spáčilová, 2000, S. 33):

\begin{tabular}{|l|l|l|}
\hline \multirow{3}{*}{ Protokoll } & Invocatio & Anrufung des göttlichen Namens \\
\cline { 2 - 3 } & Intitulatio & Angabe von Namen und Titeln des Ausstellers \\
\cline { 2 - 3 } & Inscriptio + Salutatio & Angabe von Namen und Titeln des Empfängers + Gruß \\
\hline \multirow{3}{*}{ Substantia } & Arenga & Einleitende Formel literarischen Charakters \\
\cline { 2 - 3 } & Promulgatio & Bekanntgabe des Willens des Ausstellers \\
\cline { 2 - 3 } & Narratio & Umstände, die der Rechtshandlung vorausgingen \\
\cline { 2 - 3 } & Dispositio & Darlegung des beurkundeten Rechtsgeschäfts \\
\cline { 2 - 3 } & Sanctio & $\begin{array}{l}\text { Formelhafte Anordnung weltlicher oder geistlicher Strafen für den } \\
\text { Fall einer Verletzung des Rechtsgeschäftes }\end{array}$ \\
\hline
\end{tabular}

Abb. 1: Textstrukturelemente der Textsorte Testament (vgl. Spáčilová, 2000, S. 33) 
Dabei stellt Spáčilová (2000) fest, dass Intitulatio, Narratio, Dispositio, Corroboratio und Datierung für die Olmützer Stadtkanzlei die obligatorischen Textstrukturelemente sind und dass Invocatio, Inscriptio, Arenga und Promulgatio zu den fakultativen Elementen gehören.

\section{Textlinguistische Analyse des Testaments}

An der Abschrift des Testaments von Hermann Freiherr Brunicki aus dem Jahre 1835 soll überprüft werden, welche Elemente der Textsorte Testament in dem aus Galizien stammenden Text realisiert werden. Es handelt sich hier um einen handschriftlich verfassten Text, der insgesamt 31 Seiten lang ist und größtenteils auf Deutsch geschrieben wurde. Der Text ist eine Abschrift des am 29. April 1835 aufgesetzten Testaments mit einem Stempel des Notars.

In dem untersuchten Testament werden alle oben erwähnten Elemente dieser Textsorte, jedoch in einer anderen Reihenfolge, realisiert. Da hier nur ein einziger Text untersucht wird, können keine generalisierenden Erkenntnisse über die galizienspezifischen Realisierungstypen der Textsorte Testament geäußert werden. Nichtsdestotrotz erscheint es uns wichtig, am Beispiel eines Textes Spezifika zu identifizieren, um damit auch das Interesse für die deutsche Sprache in Galizien zu wecken.

Hinsichtlich der Realisierung des Textmusters Testament kann hier festgehalten werden, dass all die von Spáčilová erwähnten Elemente der Textsorte realisiert werden. Wichtig ist, dass sich aber die jeweiligen Textstrukturelemente nicht stringent voneinander abgrenzen lassen. Das liegt auch daran, dass die Äußerungen im Text mehrfunktionalen Charakter aufweisen, was an konkreten Beispielen gezeigt wird.

Der Text beginnt auf Latein mit der Bemerkung Copia tabularis und den Registerangaben Ex lib: Instr: 498. pag: 18. - Dom: 259. pag: 390 n: b. har: [...] (S. 16). Nach diesen Angaben beginnt das eigentliche Testament, das auf Deutsch verfasst wird.

In der deutschen Sprache beginnt der Text mit der Invocatio in Form des Grußwortes „Im Namen Gottes des Vaters, des Sohnes, und des heiligen Geistes Amen“. Dem folgt ein Teil, der die Funktion der Arenga erfüllt, aber zugleich auch die Funktion der Inscriptio und Promulgatio mit realisiert, denn hier wird in pathetischer Form das Bewusstsein der Sterblichkeit sowie die Freiwilligkeit/Testierfähigkeit des Ausstellers sprachlich manifestiert.

Der Gedanke an die Vergänglichkeit eines

jeden irdischen Wesens und die Erin(n)erung ${ }^{1}$

${ }^{1}$ Die in Klammern angegebenen Buchstaben wurden von den Verfassern des Artikels hinzugefügt. Im Originaltext treten an diesen Stellen Kürzungen auf, die die Interpretation des Textes erschweren bzw. die graphematische Analyse deutlich verarmen lassen. Um die Kürzungen zu markieren, wird eine Tilde $(\sim)$ eingesetzt. 


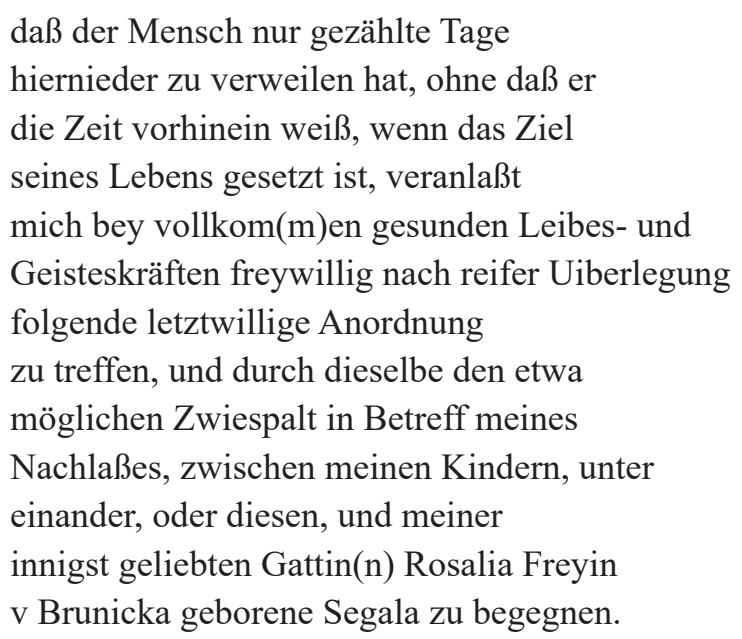

Diese Äußerung erfüllt insofern die Funktion der Promulgatio, als zum ersten Mal im Text der Wille des Testierers zum Ausdruck gebracht wird, der in weiteren Teilen konkretisiert wird. Dieser Teil des Testaments hat einen narrativen Charakter, denn es werden die Sachverhalte dargestellt, die der Rechtshandlung vorausgehen. Interessant ist hier auch die Syntax der Äußerung in Form des kanzleispezifischen Schachtelsatzes, mit der zugleich die Arenga, Inscriptio und Promulgatio realisiert werden können. Der Satz zeichnet sich jedoch durch eine relativ niedrige syntaktische Komplexität aus, denn die Nebensätze erreichen lediglich die dritte Abhängigkeitsstufe. Auffallend ist hier ein überdehntes Vorfeld mit den emotiven Inhalten sowie die Informationsdichte mit dominierendem Nominalstil sowie nachgestellter Genitiv- und Präpositionalattribuierung, die für den Kanzleistil des 18. und 19. Jahrhunderts typisch ist (vgl. u. a. Czachur, 2007).

Im nächsten Satz des Testaments werden erneut die Arenga und zugleich Promulgatio ausgeführt, indem der Testierer einerseits seine Seele Gott widmet und damit noch einmal seine Zugehörigkeit zu den Gläubigen markiert sowie andererseits den konkreten Wunsch äußert, ohne besondere Feierlichkeiten begraben zu werden:

Vor allem empfehle ich dem Allbarmherzigen,

dem Schöpfer aller Wesen meine

Seele, und äußere den Wunsch, daß mein

Körper ohne alles Gepränge zur Erde bestattet werde.

Interessant ist, dass auch im vierten Satz Teile des Protokolls wie die Inscriptio und Salutatio realisiert werden, indem hier die im vorherigen Satz nur angedeuteten Empfänger des Erlasses spezifiziert werden. Auffallend sind hier die performativen Verben segnen und empfehlen, die in einer religionsspezifischen Segensformel realisiert werden.

Ich segne alle meine vielgeliebten

Söhne Maximilian, Jacob, Moritz

Johan und Peter Freyherrn v Brunicki, dan(n) 


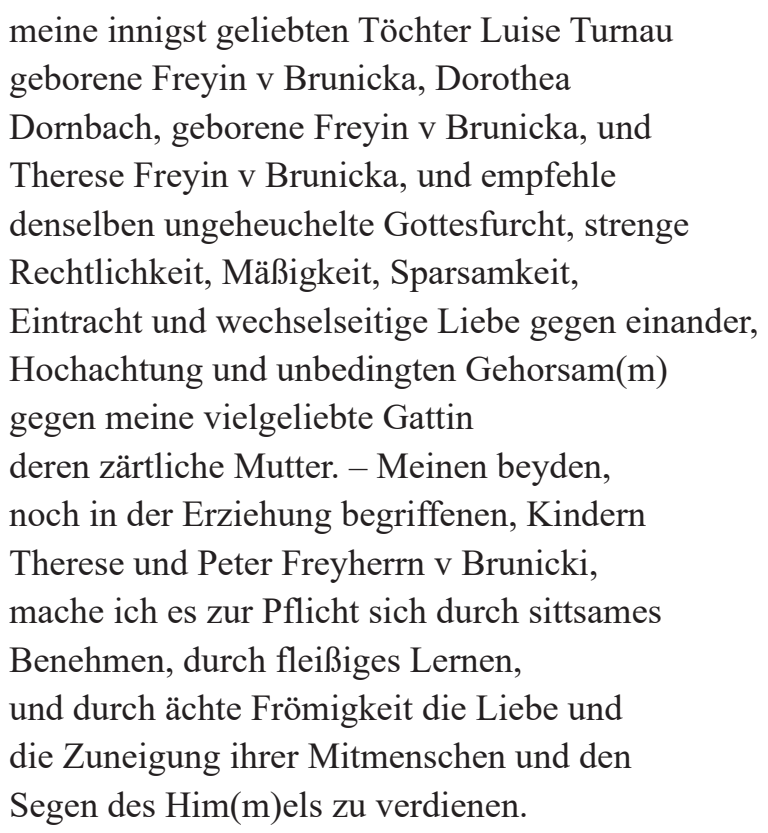

Aus diesem Grund ist es empfehlenswert, den Protokoll-Teil auch hinsichtlich der sprachlichen Beziehungsgestaltung zu untersuchen. Dies hat hier zwei Dimensionen: die persönliche Beziehung von Brunicki zu Gott als Schöpfer der geltenden Ethik und die Beziehung zu seiner Familie. Darüber hinaus weisen diese Äußerungen einen memorialen Charakter auf.

Das Testament von Brunicki hat also neben der Vermögensverwaltungsfunktion auch eine starke ethische Dimension. Er organisiert damit die Familienbeziehungen nach seinem Tod und verpflichtet seine Kinder zu einer gewissen moralischen Haltung mit einem Bezug auf aufklärungsspezifische Werte wie Gottesfurcht, Rechtlichkeit, Mäßigkeit, Sparsamkeit, Gehorsam und Liebe, wie im letzten Beispielsatz ganz deutlich wird.

Alle von Brunicki genannten Haltungen resultieren aus der christlichen/katholischen Lehre und sind eine Verpflichtung zur Einhaltung der 10 Gebote Gottes. Er erwartet also, dass seine Kinder nach seinem Tod die Lebenseinstellung fortsetzen, in der er sie erzogen hat, und ihm dadurch keine Schande machen.

In Bezug auf seine ,innigst geliebte Gattin“ äußert er auch den Wunsch an Gott, dass ,[ihr] die Allmacht eine lange Reihe von Jahren im vollen Wohlseyn angedeihen lassen wolle" (S. 18).

Dieser religiöse Kontext, die Berufung auf Gott und die moralischen Verpflichtungen der Kinder, verstärken die Majestät des letzten Willens, indem sie diesem Testament einen universellen, weit gespannten Charakter verleihen. Erblasswürdig ist in diesem Kontext nur derjenige, der eine entsprechende Lebenseinstellung hat und nach gewissen moralischen Regeln lebt.

Hochinteressant ist auch hier die sprachliche Beziehungsgestaltung in Bezug auf die Familienverhältnisse. Bezeichnungen wie meine innigst geliebte Gattin Rosalia Freyin v Brunicka geborene Segala (S. 16), alle meine vielgeliebten Söhne Maximilian, 
Jacob, Moritz, Johan und Peter Freyherrn v Brunicki (S. 16), meine innigst geliebten Töchter Luise Turnau geborene Freyin v Brunicka, Dorothea Dornbach, geborene freyin v Brunicka, und Therese Freyin v Brunicka (S. 16), meine vielgeliebte Gattin deren zärtliche Mutter (S. 16) und meine vielgeliebte Gattin (S. 29) schaffen das Bild einer glücklichen, sich liebenden und miteinander verbundenen Familie. Ersichtlich wird hier, dass diese tiefe Verbindung auch die angeheirateten Verwandten betrifft: an meinen lieben Schwiegersohn Jacob Turnau (S. 29), an meinen lieben Schwiegersohn Raymund Dornbach (S. 29).

Diese sprachlich konstruierte ethische Haltung positioniert Hermann von Brunicki einerseits als patriarchalisch den anderen Familienmitgliedern gegenüber und legitimiert ihn anderseits, den Familienmitgliedern auch ethisch orientierte Lehren bzw. Hinweise zu erteilen. Als Beweis für seine Barmherzigkeit kann auch der Hinweis auf die Tilgung der Schulden seines Bruders gelten:

Der Familie meines verewigten Bruders Joseph Freyherr v. Brunicki schenke ich anmit die mir schuldige Summe, und sehe ihr meine an ihn gehabte Schuldforderung ganz nach. (S. 28)

Das Testament von Hermann von Brunicki zeigt somit das Bild einer glücklichen, zusammenlebenden Familie, deren Mitglieder einander Hilfe leisten und das moralische Testament von Brunicki ausführen.

In den weiteren Teilen des Textes wird der letzte Wille des Ausstellers zum Ausdruck gebracht. Für die Realisierung der dispositiven Funktion des Testaments werden auch spezifische performative Verben verwendet, mit denen die Rechtshandlung vollzogen wird und deren Semantik auch in Form von Funktionsverbgefügen realisiert wird. Dominant ist hier das Verb bestimmen, das in folgenden Konstellationen vorkommt:

- „Hinsichtlich meines Vermögens bestim(m)e ich Nachstehendes“,

- „Ich bestim(m)e zugleich daß“,

- „In Betreff der Erbtheile meiner Kinder bestim(m)e ich folgendes:“,

- „Ich finde zu bestimmen, daß“,

- „so bestimme ich daß“.

Aus stilistischer Perspektive ist die vielfältige Realisierung des performativen Verbs bestimmen hinsichtlich der Valenz interessant.

- „Meiner Tochter Luise Turnau geborene Freyin v Brunicka, bestimme ich als Erbtheil aus meiner Habe...",

- ,für meine Tochter Dorothea Dornbach geborene Freyin v Brunicka bestim(m)e ich die $\operatorname{Sum}(m)$ e von...",

- „Meiner jüngsten Tochter Therese Freyinn v Brunicka fallen als Erbe von meiner Habe wegen ihrer Jugend Viertausend...".

Die Äußerungen, die als Dispositio fungieren, werden in der 1. Person Singular verwendet, darüber hinaus gibt es folgende Verben und Wortgruppen: festsetzen, auflegen, zum Vormunde einsetzen, ermächtigen, zur Pflicht machen, abtreten, sich veranlasst 
finden, übertragen. Insgesamt wird das Personalpronomen als Personaldeixis $35 \mathrm{Mal}$ verwendet, davon $21 \mathrm{Mal}$ in der performativen Funktion.

Folgende Funktionsverbgefüge werden im Text gebraucht: Anforderung treffen, Ueibereinkom(m)en treffen.

Vergleicht man diese Erkenntnisse mit den angestellten Beobachtungen von Wiktorowicz (2011) oder von Spáčilová (2000), so lässt sich feststellen, dass hier nicht anstelle des früher verwendeten performativen Verbs geben das Verb bestimmen auftritt.

Im Eschatokoll-Teil treten folgende Formulierungen auf:

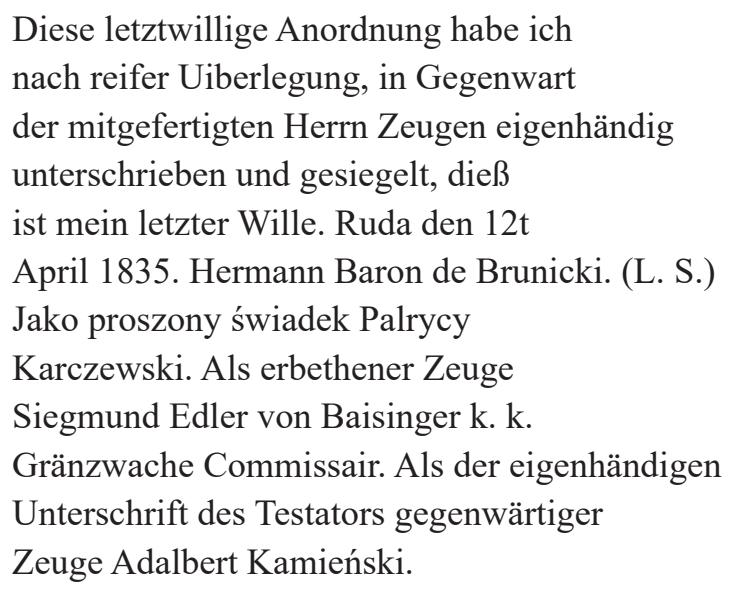

Die Illokution der jeweiligen Textstrukturelemente ist in dem Text nicht satzbezogen, sondern wird mittels unterschiedlicher komplexer Einheiten sprachlich realisiert.

\section{Die Mehrsprachigkeit und graphematische Analyse}

Das Testament von Hermann von Brunicki stellt eine interessante Mischung von drei Sprachen dar. Die leitende Sprache ist das Deutsche. Auf Deutsch wird das Testament eingeleitet:

Im Namen Gottes des Vaters, des Sohnes, und des heiligen Geistes Amen. (S. 16)

Auf Deutsch wird das Testament verfasst und teilweise auch beendet. Dies verwundert auch nicht, weil Deutsch die Muttersprache von Hermann von Brunicki war.

Neben dem Deutschen erscheinen Latein und Polnisch. Latein tritt beim Datum, 23. Novembris 1840, und bei längeren Rechtsformeln auf. Das Lateinische war seit dem Mittelalter eine offizielle, allgemein verständliche Urkundensprache, die mit der Zeit als eine traditionelle Rechtssprache anerkannt wurde. Bis heute enthält die Rechtssprache viele lateinische Wendungen und Bezeichnungen, die eine bestimmte Rechtsrealität oder ein konkretes, nicht übersetzbares Verhältnis genau bezeichnen (z. B. vacatio legis, expressis verbis). Deswegen wundert es nicht, dass in dem analysierten 
Text auch viele lateinische Fragmente und Bezeichnungen auftreten, die als bestimmte Rechtsformeln die beschriebene Situation genau bezeichnen.

Das Polnische ist begrenzt auf die Nachnamen des Erblassers und seiner Erben, auf die Ortschaftsnamen in den Grenzen des Vermögens von Hermann von Brunicki, z. B. Ruda różaniecka, Huta różaniecka, Żuki, Grochy, sowie auf eine Bemerkung am Ende der Urkunde:

Jako proszony świadek Palrycy Karczewski. (S. 29)

Diese Dreisprachigkeit resultiert aus dem Erstellungsort und aus dem Typ der Urkunde. Das Testament entstand in Galizien, das zu dieser Zeit zwar ein Teil des Kaiserreichs Österreich war, aber erst während der ersten Teilung Polens im Jahre 1772 an Österreich angeschlossen wurde. Dies bedeutet, dass die einheimische polnischsprachige Bevölkerung den Kern der Bevölkerung dieses Gebietes bildete. Daher kommen in diesem Gebiet die polnischen Ortsnamen und Nachnamen vor. Anzunehmen ist auch, dass nicht alle Vertreter der polnischen Bevölkerung zum Deutschen gewechselt hatten. Der Germanisierungsprozess dieser Gebiete verlief zwar sehr brutal, aber auch sehr schwerfällig. Es kann nicht ausgeschlossen werden, dass es 68 Jahre nach der ersten Teilung Polens immer noch Leute gab, die kein Deutsch sprachen. Die Behördenvertreter mussten diese Tatsache einfach berücksichtigen und sich der Realität anpassen. Dies verursachte, dass Polnisch in offiziellen Urkunden aller Art präsent war (vgl. Fellerer, 2005).

In der analysierten Urkunde weisen die Sprachen prinzipiell keinen gegenseitigen Einfluss auf. Für den Einfluss einer Sprache (das Polnische) auf eine andere (das Deutsche) gibt es nur einen einzigen Beleg:

Dreytausend sieben hundert fünfzig Dukaten oder siebzehn tausend acht hundert siebenzig fünf Gulden Conventions-Münze. (S. 23)

Auffallend ist in diesem Falle eine andere Vorgehensweise bei der Bildung der Kardinalia. Während im Deutschen die Zahl 17875 wörtlich als siebzehntausendachthundertfünfundsiebzig dargestellt wird (d. h. die Einer werden vor die Zehner gestellt und mit einem Verbindungselement $u n d$ verbunden), kommt im Text ein dem Deutschen fremdes Kardinaliabildungsvorgehen vor: [...] siebenzig fünf. Diese Vorgehensweise deutet auf den polnischen Einfluss hin.

Als eine traditionelle, stark formalisierte Urkunde weist das Testament viele rechtliche Eigenschaften auf. Als eine Rechtsurkunde fügt sich das Testament in eine sui generis Landschaft rechtlicher Texte ein und unterliegt konkreten, damit verbundenen Voraussetzungen, zu denen u. a. die höchstpräzise Sprache gehört. Im analysierten Text finden sich lateinische Wendungen wie cum attinentiis (S. 26, 27), mit Zubehör“" (Einnahmes)quitten (S. 27), Administrator (S. 29). Auf Latein werden die Daten, z. B. 
Nov $\sim$ r s (S. 16), die Titelbemerkung Copia Tabularis (S. 16), die Kürzungen beim Register am Anfang der Urkunde: Ex lib: Instr: 498. pag: 18. - Dom: 259. pag: 390 n: b. har: [...] (S. 16) und eine längere Textpassage am Ende des Textes (S. 29 f.) angegeben.

In graphematischer Hinsicht vertritt das Testament von Hermann von Brunicki die klassische Kurrentschrift. Es lassen sich zwei unterschiedliche Schriftcharakter unterscheiden. Die Seiten 16-22 wurden von einer anderen Person geschrieben als die Seiten 23-30. Diese Unterschiede betreffen die Größe der Grapheme sowie ihren Neigungswinkel. Darüber hinaus ist die Schrift des Schreibers 2 etwas schwieriger zu entziffern.

Der Tradition nach werden alle fremdsprachigen Wörter mit lateinischen Buchstaben geschrieben. Dies bedeutet, dass alle lateinischen Rechtsformeln und Wendungen, sowie alle polnischen Eigennamen in einer anderen Schrift verfasst wurden als der Rest des Textes.

Die vorkommenden Grapheme weichen von einem Kurrentschriftmuster nicht wesentlich ab.

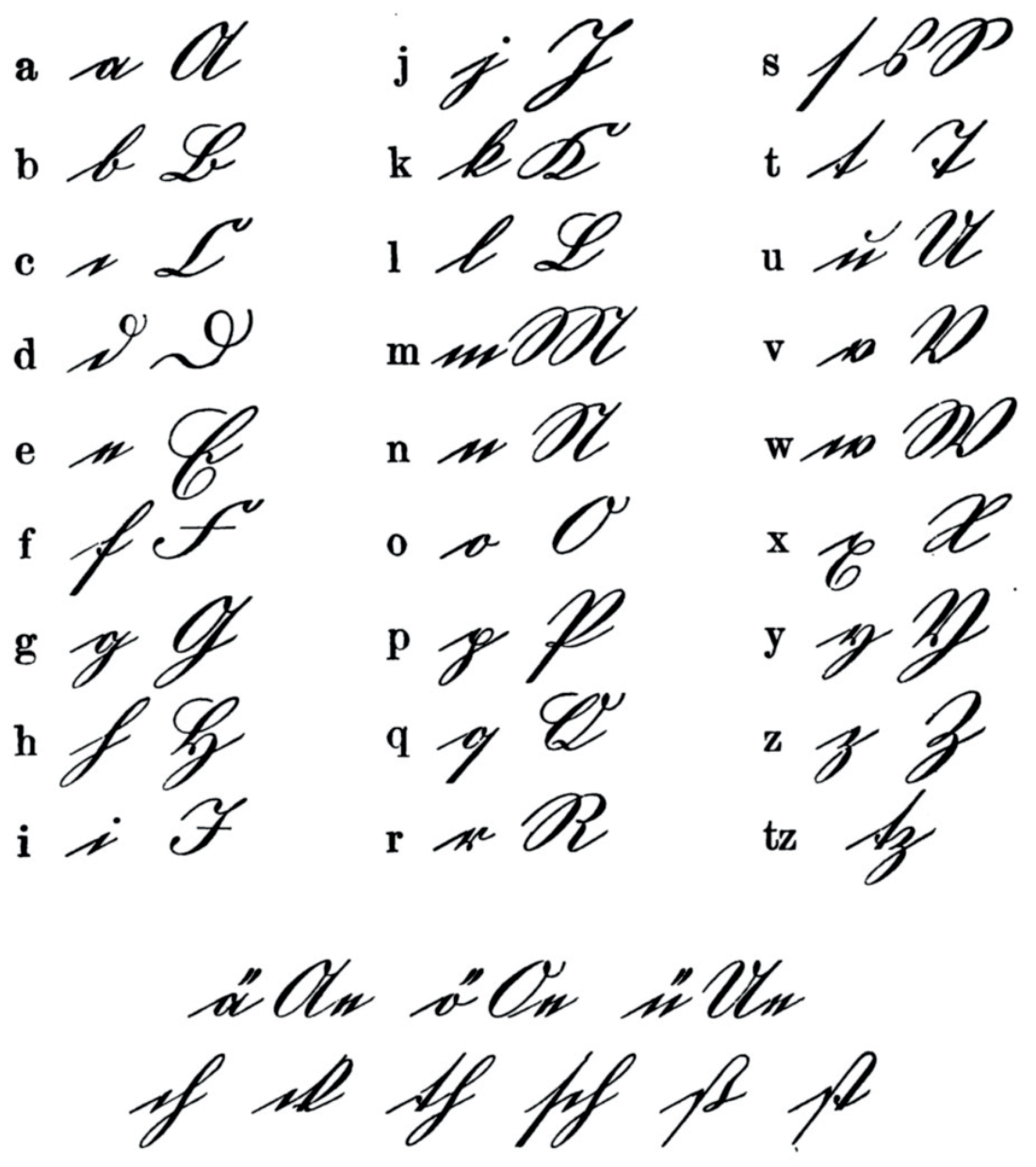

Abb. 2: Die Zusammenstellung der deutschen Kurrentschrift und der entsprechenden Grapheme der lateinischen Schrift 
Die Unterschiede zwischen den im Text gebrauchten Graphemen und dem Muster lassen sich meistens auf den individuellen Schriftcharakter zurückführen. Der einzige systematische Unterschied betrifft das Graphem $<\mathrm{Ui}>$, das konsequent in dieser Form erscheint. Diese Konsequenz erstreckt sich auch auf andere Urkunden aus dem Brunicki-Archiv.

Sehr typisch für den analysierten Text sind auch unterschiedliche Kürzungen, die besonders häufig bei $<\mathrm{nn}>$ und $<\mathrm{mm}>$ erscheinen. Die Kürzungen kommen sowohl

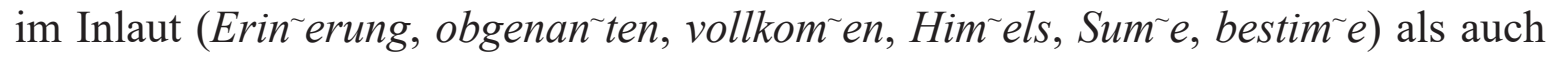
im Auslaut (Gatin , Johan $\sim$, kan ${ }^{\sim}$, dan ) vor. Nur einmal erscheinen die Kürzungen bei $<\mathrm{a}>$ : ba re (S. 17) und bei $<\mathrm{rr}>$ : Freyhe (S. 30). Häufig sind auch Kürzungen, die zwei oder mehrere Grapheme ersetzen, z. B. $4^{t \sim s}$ oder Con: Mze (= Conventionsmünze). Interessanterweise treten die Kürzungen nur bei Schreiber 1 auf. Schreiber 2 scheint beim Schreiben fleißiger gewesen zu sein. Die gebrauchten Kürzungen können aus einer bestimmten Hektik beim Niederschreiben des mündlich diktierten Textes resultieren.

Da sich die benutzte Schrift nicht wesentlich von dem Kurrentschriftmuster unterscheidet, werden im Folgenden nur die Abweichungen besprochen.

$<\mathrm{Ui}>=<\ddot{\mathrm{U}}>$

Der auffallendste Unterschied betrifft das Graphem $<\mathrm{Ui}>=<\ddot{\mathrm{U}}>$. Da es kein besonderes Zeichen für die Majuskel des U-Umlauts gab, versuchte man diesen unterschiedlich wiederzugeben. Als Muster galt das Graphem $<\mathrm{Ue}>$. In den Urkunden des Brunicki-Archivs erscheint jedoch systematisch das Graphem $<\mathrm{Ui}>$. Diese Schreibweise ist nicht auf einen konkreten Schreiber begrenzt. Daraus kann man den Schluss ziehen, dass es eine Eigenschaft der galizischen Kanzlei war. Es fehlen jedoch entsprechende Untersuchungen und eine solche Bemerkung sollte man nur als eine genauer zu untersuchende Hypothese betrachten. Das Graphem $<\mathrm{Ui}>$ erscheint zweimal: nach reifer Uiberlegung (S. 16) und vom Tage der Uibernahme des oberwähnten Gutes (S. 26). Im Unterschied dazu kommt bei der Minuskel nur das Graphem < $\ddot{u}>$ vor (über, Eigenthümer, unterstützen, führenden, müßen).

$<\ddot{\mathrm{a}}>=<\mathrm{e}>$

Das Graphem $<\ddot{a}>$ erscheint sowohl in den durch Schrifttradition sanktionierten Positionen als auch an den Stellen, an denen $<\mathrm{e}>\mathrm{zu}$ erwarten wäre: ächte (S. 17), Gutdänken (S.17), Gränzhaufen (S. 24), Gränze (S. 25). Diese überraschende Schreibweise tritt bei beiden Schreibern auf.

$<\mathrm{y}>=<\mathrm{i}>$

Das Graphem $<\mathrm{y}>$, das ein $<\mathrm{i}>$ ersetzt, hat eine lange Tradition und reicht bis zum Anfang der schriftlichen Fixierung zurück. Die Häufigkeit dieses Graphems hat mit der Zeit abgenommen. Die alte Schreibweise $<$ ey $>$ für $<$ ei $>$ erscheint im Text relativ selten. 
Es lässt sich eine gewisse Regel beobachten - die Schreibung < ey > tritt vor allem in den Zusammensetzungen mit bei- und frei und bei einigen Wörtern auf:

- bey (S. 16), beystand (S. 17), beyläufig (S. 19), Herbeyschaffung (S. 27), beyzustellen (S. 27), beyzuschaffen (S. 27), beygestellte (S. 27)

- freyes (S. 28), freywillig (S. 16), Freyinn (S. 16, 28, 29, 30), Freyherr (S. 16, 28)

- beyden (S. 16, 23)

- $\operatorname{drey(S.~20,21),~dreyzehntausend~(S.~21)~}$

- zweyhundert (S. 22), zweytausend (S. 22)

- Wohlseyn (S. 18).

$<\beta>$

Das Graphem $<\beta>$ erscheint dort, wo man es mit Doppel-s zu tun hat. Die Schreibung $<\beta>$ erscheint sowohl nach den kurzen Vokalen: $d a \beta$ (S. 25, 26, 27, 28, 29), veranlaßt (S. 16), Nachlaßes (S. 16), Nachlaßens (S. 29), beßer (S. 20), müßen (S. 21), hinterlaßenen (S. 23), beseßen (S. 23), Ausschluß (S. 24), zulaßen (S. 28), als auch nach den langen Vokalen: überdieß (S. 17), standesmäßige (S. 21), sowie nach Diphthongen: weiß (S. 16), fleißiges (S. 16).

Überraschend ist die Schreibung Waßerdurchleitung (S. 25), die allen phonetisch-graphematischen Regeln widerspricht. Das Graphem $<\beta>$ ist, sprachhistorisch gesehen, eine Ligatur aus zwei unterschiedlichen s-Graphemen: dem \ und dem 3, die seit dem 16. Jh. zu dem heute verwendeten Zeichen zusammengewachsen waren, jedoch nur in den Positionen, in denen im Mittelhochdeutschen die oben erwähnten Grapheme auftraten. Das Wort Wasser wurde immer mit dem Graphem -33- geschrieben (ahd. wazzar, mhd. wazzer), das sich zur heutigen Schreibung -ss- entwickelte. Bemerkenswert ist auch, dass das mhd. Graphem , 3 “ den phonetischen Wert von [s] hatte.

Interessanterweise erscheint auch einmal die Form verhassen (S. 28), bei der die zu erwartende Schreibung mit $\langle\beta>$ doch durch Doppel-s ersetzt wurde. Diese Inkonsequenz kann daraus resultieren, dass es zu dieser Zeit noch keine allgemeingültige Regulierung für die Rechtschreibung gab. Da keine orthographischen Regeln galten, herrschte eine gewisse Willkür bei der Schreibung.

$<\mathrm{th}>=<\mathrm{t}>$

Das Graphem $<$ th $>$ kommt zwar im heutigen Deutsch vor, aber wird auf Fremdwörter begrenzt. Im 19. Jh. war diese Schreibung in Mode. Man kann ihr auch keine zusätzliche phonetische Qualität zuschreiben, weil es kein Zeichen für Aspiration war. Im analysierten Text tritt die Schreibung $<$ th $>$ oft in Erscheinung und zwar im einheimischen deutschen Wortschatz und in Eigennamen (Dorothea, Therese). Sie erscheint sowohl im Anlaut (Theil) als auch im Inlaut (Eigenthum, Eigenthümer, Waldbestandtheile, Wirthschaft, Antheil, betheilen, Erbtheil, Waldtheil, Gutstheils, Pflichttheil, Viehenantheile, Wiesenantheil, nothwendig, nöthige, vorräthig, vortheilhaft, Wirthshäuser, erbethener) und im Auslaut (Heirath). 
$<\mathrm{c}>=<\mathrm{k}>$

Das Graphem $<\mathrm{k}>$ setzte sich lange in der deutschen Schrifttradition durch, vor allem deswegen, weil es dem Lateinischen fremd war. Sehr lange wurde das Graphem $<\mathrm{c}>$ für den Laut [k] gebraucht, bis sich die Regel herausgebildete, dieses Graphem in den neueren Entlehnungen zu verwenden. Mitte des 19. Jhs. war es noch nicht klar, wann beide Grapheme gebraucht werden sollten, deswegen findet man im Text oft Belege für eine unterschiedliche Schreibung der gleichen Wörter. Auffallend ist, dass das Graphem <c> vor allem in Fremdwörtern auftritt: Jacob, Fundus instructus, October, Con. M ze (Conventions-Münze), Conventions-Münze, cum attientiis, Acrar, Commissair. Daneben erscheint auch sporadisch die Schreibung der Fremdwörter mit $<\mathrm{k}>$ : Aktivforderungen, Konv: Mze, Konventions-Münze. Im einheimischen Wortschatz kommt die Schreibung < k> vor: Kinder, Gutdänken, Urkunde, Ankaufe, Kreise, Dukaten.

\section{Zusammenfassung}

Das Testament von Brunicki stellt ein höchst interessantes, wissenschaftlich noch nicht untersuchtes Textmuster des galizischen Deutsch dar. Es liefert auch eine wertvolle Grundlage für die Erforschung der Sprachgeschichte des 19. Jahrhunderts, die vor allem als Mentalitätsgeschichte aufzufassen ist. Die analysierte Textsorte Testament wird sprachlich und konzeptionell anders realisiert als die in der Krakauer oder Olmützer Kanzlei. Auf den ersten Blick ist die Komplexität auffallend, die auch eine scharfe Trennung der jeweiligen Textstrukturelemente schwer macht. Die für die jeweiligen Textstrukturelemente spezifischen Propositionen, die sowohl im 15.-16. Jahrhundert als auch im 19. Jahrhundert vergleichbar sind, werden allerdings satzübergreifend realisiert, so dass wir es mit mehrdimensionalen Handlungskomplexen zu tun haben. Einige Unterschiede lassen sich auch hinsichtlich der syntaktischen Strukturen feststellen. Zwar ist im galizischen Testament die Spezifik des Kanzleistils erkennbar, aber die syntaktischen Strukturen sind komplexer. Neben der deontischen Funktion einiger Strukturen, mit denen konkrete Rechtshandlungen vollzogen werden, wird hier auf die emotionale Ebene Wert gelegt, indem sich der Testierer in Beziehung zu Gott einerseits und zu seinen Familienmitgliedern andererseits emotionsgeladen sprachlich positioniert. Somit ist das Testament von Hermann von Brunicki ein Zeugnis des schöpferischen Denkens der vergangenen Epoche.

Interessant wäre aber in diesem Zusammenhang der Vergleich zum einen der Textsorte Testament im galizischen Deutsch mit anderen Urkunden dieser Zeit und zum anderen mit denselben Textsorten aus Gebieten z. B. von Preußen, um somit die Spezifik des galizischen Sprachgebrauchs im Rechtsbereich erfassen zu können. 


\section{Literatur}

Becker-Mrotzek, M. (1999). Die Sprache der Verwaltung als Institutionensprache. In L. Hoffmann \& H. Kalverkämper \& H.E. Wiegand (Hrsg.), Fachsprachen. Bd. 2 (S. 1391-1402). Berlin: de Gruyter. Becker-Mrotzek, M. \& Scherner, M. (2000). Textsorten der Verwaltung. In K. Brinker \& G. Antos \& W. Heinemann \& F.S. Sager (Hrsg.), Text- und Gesprächslinguistik. 1. Halbband (S. 628-641). Berlin: de Gruyter.

Besier, G. \& Stokłosa, K. (2015). Polen und Ruthenen im habsburgischen Galizien während der ersten Hälfte des 19. Jahrhunderts. In A. Hanus \& R. Büttner (Hrsg.), Galizien als Kultur- und Gedächtnislandschaft im kultur- und sprachwissenschaftlichen Diskurs (S. 19-38). Frankfurt am Main: Peter Lang.

Bieberstedt, A. (2007). Textstruktur - Textstrukturvariation - Textstrukturmuster. Lübecker mittelniederdeutsche Testamente des 14. und 15. Jahrhunderts. Wien: Praesens.

Bieberstedt, A. (2009). Strukturmuster in der Textsorte Testament. Dargestellt am Beispiel Lübecker Bürgertestamente des 14. und 15. Jahrhunderts. In P. Ernst (Hrsg.), Kanzleistil: Entwicklung, Form, Funktion. Beiträge zur 4. Tagung des Internationalen Arbeitskreises Kanzleisprachenforschung, Wien, 24. und 25. November 2006 (S. 9-56). Wien: Praesens.

Biszczanik, M. (2013). Die ältesten Stadtbücher von Sprottau/Szprotawa. Ein Beitrag zur Erforschung des Frühneuhochdeutschen in Niederschlesien. Zielona Góra: Oficyna Wydawnicza Uniwersytetu Zielonogórskiego.

Bogacki, J. (2009). Graphematische Untersuchungen zum Vokalismus im deutschsprachigen Kanzleischrifttum des 15. und 16. Jahrhunderts aus Namslau, Brieg, Neisse und Leobschütz. Berlin: Trafo.

Chromik, G. (2010). Schreibung und Politik. Untersuchungen zur Graphematik der frühneuhochdeutschen Kanzleisprache des Herzogtums Teschen. Kraków: Wydawnictwo Uniwersytetu Jagiellońskiego.

Cwanek-Florek, E. (2015). Deutsche Entlehnungen in der polnischen Amtssprache Galiziens am Beispiel ausgewählter „Akten der Stadt Rzeszów”. In E. Cwanek-Florek \& I. Nöbauer (Hrsg.), Sprachliche Wechselbeziehungen in der Habsburgermonarchie (S. 85-106). Wien: Polnische Akademie der Wissenschaften. Wissenschaftliches Zentrum in Wien.

Cwanek-Florek, E. (2018). Zu Tendenzen der Einwirkung des Deutschen auf die polnische Verwaltungssprache Galiziens am Beispiel der deutschen Komposita und ihrer Entsprechungen im Polnischen im Allgemeinen bürgerlichen Gesetzbuch (ABGB) (1811) / Księga Ustaw Cywilnych (1811). Studia Niemcoznawcze, LXI, 851-864.

Czachur, W. (2007). Textmuster im Wandel. Ein Beitrag zur textlinguistischen Erforschung der Vereinssatzungen im 19. Jahrhundert. Wrocław-Dresden: ATUT.

Czachur, W. (2008). Die Analyse der Vereinssatzungen des 19. Jahrhunderts vor dem Hintergrund der pragmatisch und kognitiv orientierten Sprachgeschichte. In W. Czachur \& M. Czyżewska (Hrsg.), Vom Wort zum Text. Studien zur deutschen Sprache und Kultur. Festschrift für Józef Wiktorowicz zum 65. Geburtstag (S. 461-474). Warszawa: Instytut Germanistyki UW.

DUDEN. (2006). Deutsches Universalwörterbuch. Mannheim-Leipzig-Wien-Zürich: Dudenverlag.

Fellerer, J. (2004). Sprache und Politik: Das galizische Verwaltungswesen (1772-1914). Österreichische Osthefte, 46, 1/2, 51-90.

Fellerer, J. (2005). Mehrsprachigkeit im galizischen Verwaltungswesen (1772-1914). Eine historisch-soziolinguistische Studie zum Polnischen und Ruthenischen (Ukrainischen). Köln-Weimar: Böhlau Verlag.

Feret, A. (2013). Zur Sprache der Werbeanzeigen in den Zeitschriften der k. k. Kreisstadt Rzeszów in der Zeit von 1890 bis 1910. tekst i dyskurs - text und diskurs, 6, 373-389.

Feret, A. (2014). Zu Lehnbildungen mit der deutschen Basis in der polnischen Behördensprache von Rzeszów, Sanok und Tarnobrzeg am Anfang des 20. Jahrhunderts. Germanica Wratislaviensia, 139, 141-152.

Grabarek, J. (1984). Die Sprache des Schöffenbuches der Alten Stadt Toruń. Rzeszów: Wydawnictwo WSP Rzeszów. 
Hanus, A. \& Büttner, R. (Hrsg.). (2015). Galizien als Kultur- und Gedächtnislandschaft im kultur- und sprachwissenschaftlichen Diskurs. Frankfurt am Main: Peter Lang.

Hoffmann, L. (1989). Rechtsdiskurse. Tübingen: Narr.

Kaleta-Wojtasik, S. (2001). Mittelalterliches Testament als Textsorte. Versuch einer Untersuchung anhand deutschsprachiger Testamente der Krakauer Bürger aus dem XV. Jh. In A. Schwarz \& L.L. Abplanalp (Hrsg.), Textallianzen am Schnittpunkt der germanistischen Disziplinen (S. 259-272). Frankfurt am Main: Peter Lang.

Kaleta-Wojtasik, S. (2004). Graphematische Untersuchungen zum Codex Picturatus von Balthasar Behem. Kraków: Wydawnictwo Uniwersytetu Jagiellońskiego.

Malinowska, E. (2001). Wypowiedzi administracyjne - struktura i pragmatyka. Opole: Wydawnictwo Uniwersytetu Opolskiego.

Napierała, K. \& Plesiński, W. (2013). Lexikon des polnischen Rechts. Warszawa: C.H.BECK.

Paul, H. (1992). Deutsches Wörterbuch. 9., vollständig neu bearbeitete Auflage von Helmut Henne und Georg Objartel unter Mitarbeit von Heidrun Kämper-Jensen. Tübingen: de Gruyter.

Pfeifer, W. (1993). Etymologisches Wörterbuch des Deutschen, Bd. 2 (M-Z), 2. Auflage, durchgesehen und ergänzt von Wolfgang Pfeifer. Berlin: de Gruyter.

Piętkowa, R. (2000). Gatunki stylu urzędowego - wzorce i realizacja. In D. Ostaszewska (Hrsg.), Gatunki mowy i ich ewolucja. Band 1: Mowy piękno wielorakie (S. 96-108). Katowice: Wydawnictwo Uniwersytetu Śląskiego.

Rzeszutko, M. (2003). Rozprawa sądowa w świetle lingwistyki tekstu. Lublin: Wydawnictwo Uniwersytetu Marii Curie-Skłodowskiej.

Spáčilová, L. (2000). Deutsche Testamente von Olmützer Bürgern: Entwicklung einer Textsorte in der Olmützer Stadtkanzlei in den Jahren 1416-1566. Wien: Praesens.

Wiktorowicz, J. (2011). Krakauer Kanzleisprache. Forschungsperspektiven und Analysemethoden. Warszawa: Instytut Germanistyki UW.

Wiktorowicz, J. (2012). Die deutsche Kanzleisprache in Polen. In A. Greule \& J. Meier \& A. Ziegler (Hrsg.), Kanzleisprachenforschung: Ein internationales Handbuch (S. 599-607). Berlin-Boston: de Gruyter.

Wojtak, M. (2004). Gatunki urzędowe na tle innych typów piśmiennictwa użytkowego - zarys problematyki. In E. Malinowska (Hrsg.), Język - prawo - społeczeństwo (S. 131-141). Opole: Wydawnictwo Uniwersytetu Opolskiego.

Wyrwas, K. (2002). Skarga jako gatunek mowy. Katowice: Wydawnictwo Uniwersytetu Śląskiego.

Żmigrodzka, B. (1997). Testament jako gatunek tekstu. Katowice: Wydawnictwo Uniwersytetu Śląskiego. 